\title{
Replication of Reduced Pattern Electroretinogram Amplitudes in Depression With Improved Recording Parameters
}

\author{
Evelyn B. N. Friedel ${ }^{1,2,3 \dagger}$, Ludger Tebartz van Elst ${ }^{1 * \dagger}$, Céline Schmelz ${ }^{4}$, Dieter Ebert ${ }^{1}$, \\ Simon Maier ${ }^{1}$, Dominique Endres ${ }^{1}$, Kimon Runge ${ }^{1}$, Katharina Domschke ${ }^{1,5}$, \\ Emanuel Bubl ${ }^{1}$, Jürgen Kornmeier ${ }^{1,6}$, Michael Bach ${ }^{2}$, Sven P. Heinrich ${ }^{2}$ and \\ Kathrin Nickel ${ }^{1}$ \\ ${ }^{1}$ Department of Psychiatry and Psychotherapy, Medical Center-University of Freiburg, Faculty of Medicine, University of \\ Freiburg, Freiburg, Germany, ${ }^{2}$ Eye Center, Medical Center-University of Freiburg, Faculty of Medicine, University of \\ Freiburg, Freiburg, Germany, ${ }^{3}$ Faculty of Biology, University of Freiburg, Freiburg, Germany, ${ }^{4}$ Pfalzklinikum - Clinic for \\ Psychiatry and Neurology, Klingenmünster, Germany, ${ }^{5}$ Center for Basics in Neuromodulation, Faculty of Medicine, University \\ of Freiburg, Freiburg, Germany, ${ }^{6}$ Institute for Frontier Areas of Psychology and Mental Health, Freiburg, Germany
}

OPEN ACCESS

Edited by: Jason C. Park,

University of Illinois at Chicago, United States

Reviewed by: Jan Kremers,

University Hospital Erlangen, Germany Shresta Patangay, University of Illinois at Chicago, United States

*Correspondence: Ludger Tebartz van Elst tebartzvanelst@uniklinik-freiburg.de

†These authors share first authorship

Specialty section: This article was submitted to Ophthalmology a section of the journal Frontiers in Medicine

Received: 28 June 2021 Accepted: 06 October 2021 Published: 29 October 2021

Citation:

Friedel EBN, Tebartz van Elst L, Schmelz C, Ebert D, Maier $S$, Endres D, Runge K, Domschke K, Bubl E, Kornmeier J, Bach M, Heinrich SP and Nickel K (2021) Replication of Reduced Pattern Electroretinogram Amplitudes in Depression With Improved Recording Parameters. Front. Med. 8:732222. doi: 10.3389/fmed.2021.732222
Background: The retina has gained increasing attention in non-ophthalmological research in recent years. The pattern electroretinogram (PERG), a method to evaluate retinal ganglion cell function, has been used to identify objective correlates of the essentially subjective state of depression. A reduction in the PERG contrast gain was demonstrated in patients with depression compared to healthy controls with normalization after remission. PERG responses are not only modulated by stimulus contrast, but also by check size and stimulation frequency. Therefore, the rationale was to evaluate potentially more feasible procedures for PERG recordings in daily diagnostics in psychiatry.

Methods: Twenty-four participants (12 patients with major depression (MDD) and 12 age- and sex-matched healthy controls) were examined in this pilot study. We investigated PERG amplitudes for two steady-state pattern reversal frequencies $(12.5 / 18.75 \mathrm{rps})$ and four sizes of a checkerboard stimulus $\left(0.8^{\circ}, 1.6^{\circ}, 3.2^{\circ}\right.$, and $\left.16^{\circ}\right)$ to optimize the PERG recordings in MDD patients.

Results: Smaller PERG amplitudes in MDD patients were observed for all parameters, whereby the extent of the reduction appeared to be stimulus-specific. The most pronounced decline in the PERG of MDD patients was observed at the higher stimulation frequency and the finest pattern, whilst responses for the largest check size were less affected. Following the PERG ratio protocol for early glaucoma, where similar stimulus dependent modulations have been reported, we calculated PERG ratios $\left(0.8^{\circ} / 16^{\circ}\right)$ for all participants. At the higher frequency (18.75 rps), significantly reduced ratios were observed in MDD patients.

Conclusion: The "normalization" of the PERG responses - via building a ratio - appears to be a very promising approach with regard to the development of an objective biomarker of the depressive state, facilitating inter-individual assessments of PERG recordings in patients with psychiatric disorders.

Keywords: pattern electroretinogram, PERG, depression, check size, dopamine 


\section{INTRODUCTION}

As an ontogenetic part of the brain, the retina exhibits high levels of many neurotransmitters of the central nervous system, including dopamine (1). Since the retina represents a more accessible structure than the brain itself for related measurements, it recently gained increasing attention in other, non-ophthalmological research fields, such as neurology or psychiatry (2).

Indeed, previous studies indicate alterations in visual processing in diseases which are associated with a disturbance in the central dopaminergic homeostasis. These include Parkinson's disease $(3-5)$, schizophrenia $(6,7)$ and major depressive disorder (MDD) (8-10).

Bubl et al. (8) initially reported higher contrast detection thresholds in patients suffering from MDD. In further research, they took advantage of a more objective electrophysiological approach from ophthalmology, the pattern electroretinogram (PERG), to demonstrate objective correlates of the essentially subjective state of depression (9).

The electroretinogram (ERG) uses corneal electrodes to measure the electrical activity of the retina in response to visual stimulation (11). The PERG is mostly generated by the retinal ganglion cells (12) which are stimulated by local contrast changes in black/white reversing pattern stimuli, like checkerboards (13, 14). The PERG allows both, an assessment of the macular function and a direct measurement of the retinal ganglion cell integrity (15). Therefore, it is-so far-primarily applied in ophthalmology for detecting early glaucomatous dysfunction (16-18).

In recent years, PERG has become increasingly important in psychiatric research as a possibility to map the integrity of the cerebral dopaminergic system indirectly via retinal ganglion cell function with minimal invasiveness (3, 19-22).

Bubl et al. (9), for instance, observed a remarkable reduction in the PERG contrast gain (corresponding to the increase in amplitude with ascending stimulus contrast) of about 50\% in patients with MDD compared to healthy controls, with a significant negative correlation of contrast gain with depression severity. Moreover, with remission of the depressive symptoms, a normalization of the reduced retinal signals was observed (10). Therefore, it was postulated that the PERG could be a meaningful measurement tool for psychiatric disorders with the contrast gain as a state marker for depression (10). Table $\mathbf{1}$ lists preliminary and present investigations focusing on the contrast sensitivity and the PERG-based contrast gain in MDD patients.

The International Society for Clinical Electrophysiology of Vision (ISCEV) published the current PERG standards in 2012 with recommendations for measurement parameters, calibrations and settings of PERG recordings (14). Various parameters have to be considered when recording the PERG signal.

For the standard PERG, a symmetrical black/white reversing checkerboard pattern with a constant mean luminance should be presented at a standard $\left(15^{\circ}\right)$ or large field size $\left(\approx 30^{\circ}\right)$. A check size of $0.8^{\circ}\left( \pm 0.2^{\circ}\right)$, a reversal rate of approximately $16 \mathrm{rps}$ $(8 \mathrm{~Hz}) \pm 20 \%$ (for steady state stimulation) and a high stimulus contrast (> 80\%) is recommended in the current guidelines (14). As the PERG amplitude increases almost linearly with increasing stimulus contrast (13), the PERG contrast gain can be calculated from a linear regression line (PERG contrast transfer function) as described by Bubl et al. (9). The slope of this regression line is modulated by the stimulus frequency as well as by the check sizes presented. Higher frequencies lead to a steeper slope of the PERG contrast transfer function, whereas the use of larger patterns $(\approx$ $\left.4^{\circ}\right)$ seems to counteract this effect (25).

\section{Aims of the Study}

The recommended standard recording parameters of the ISCEV (14) for clinical PERG assessment have been adapted for ophthalmologic patients. However, it has not yet been investigated whether they are equally suitable for PERG recordings in psychiatric patients.

The aim of the current study was (1) to replicate the findings of reduced PERG responses in patients with depression $(9,10)$ in an independent sample and (2) to improve the PERG protocols for this patient group with a specific focus on check size and stimulus frequency.

\section{Check Size}

It is known that the PERG amplitudes attenuate with poor visual acuity (26). Proper refraction is thus mandatory for PERG recordings, but difficult to implement in non-ophthalmological settings. This effect can be bypassed by using very coarse checkerboard patterns for the stimulation, which are clearly above the visual acuity threshold. In the present study, we investigated the PERG in response to a whole set of black/white reversal checkerboards with the following check sizes: $0.8^{\circ}, 1.6^{\circ}$, $3.2^{\circ}$, and $16^{\circ}$.

\section{Frequency}

In previous studies about PERG effects in patients with depression, a stimulation frequency of 12.5 rps was applied. Higher reversal frequencies have been reported to be capable to increase the sensitivity for detecting ophthalmological diseases like glaucoma $(27,28)$. In the present study, we compared PERG amplitudes for two steady-state frequencies for pattern reversals (12.5 and $18.75 \mathrm{rps})$ to assess whether they can be applied equivalently.

\section{MATERIALS AND METHODS}

\section{Participants}

The study was approved by the ethics committee of the University Medical Center Freiburg (Approval ID: 93/04) and was conducted in accordance with the Declaration of Helsinki. All participants gave their written informed consent. Patients were recruited at the Department of Psychiatry and Psychotherapy, University of Freiburg. The diagnosis of a major depressive episode was established by an experienced specialist in psychiatry according to DSM-5 criteria. A depressive episode in the context of bipolar disorder, the presence of psychotic symptoms, and comorbid alcohol abuse were defined as exclusion criteria. Initially, 17 patients with a diagnosis of major depressive 
TABLE 1 | Previous studies on contrast sensitivity and/or PERG responses in patients with major depression.

\begin{tabular}{|c|c|c|c|c|}
\hline References & $N$ (Patients/HC) & $\begin{array}{l}\text { Age (years) mean } \\
\text { (SD) }\end{array}$ & $\begin{array}{l}\text { Measurement parameters (e.g., pattern } \\
\text { size, reversal rate, contrast level) }\end{array}$ & Results (Patients/HC) \\
\hline 1. Bubl et al. (8) & $\begin{array}{l}28 \mathrm{MDD} \\
21 \mathrm{HC}\end{array}$ & $\begin{array}{l}31.8(9.5) \\
33.1(10)\end{array}$ & $\begin{array}{l}\text { Gabor patches: } \\
\text { Size: } 2 \text { cpd } \\
\text { Contrasts: 1, 3, 10, 20, 30, 40, 50\% }\end{array}$ & $\begin{array}{l}\text { Elevated contrast discrimination } \\
\text { threshold in MDD }\end{array}$ \\
\hline $\begin{array}{l}\text { 2. Bubl et al. (9) [see } \\
\text { technical note in Bubl } \\
\text { et al. (23)] }\end{array}$ & $\begin{array}{l}40 \mathrm{MDD} \\
\text { (20 medicated, } \\
20 \text { without medication) } \\
40 \mathrm{HC}\end{array}$ & $\begin{array}{l}43.2(6.3) \\
44.6(4.5) \\
41.8(4.5) \\
43.3(6.3)\end{array}$ & $\begin{array}{l}\text { PERG } \\
\text { Check size: } 0.51^{\circ} \\
\text { Reversal rate: } 12.5 \mathrm{rps} \\
\text { Contrasts: } 3.2,7.3,16.2,36,80 \%\end{array}$ & $\begin{array}{l}\text { Reduced PERG contrast gain in } \\
\text { MDD ( } \sim 50 \% \text { reduction in MDD) }\end{array}$ \\
\hline 3. Bubl et al. (10) & $\begin{array}{l}14 \text { MDD } \\
\text { (10 remitted, } \\
4 \text { not remitted) } \\
40 \mathrm{HC}\end{array}$ & $\begin{array}{l}40.3(12.8) \\
48.8(9.8) \\
43.3(12.7)\end{array}$ & $\begin{array}{l}\text { PERG } \\
\text { Check size: } 0.51^{\circ} \\
\text { Reversal rate: } 12.5 \mathrm{rps} \\
\text { Contrasts: } 3.2,7.3,16.2,36,80 \%\end{array}$ & $\begin{array}{l}\text { Normalization of reduced } \\
\text { PERG-based contrast gain in } \\
\text { MDD with remission }\end{array}$ \\
\hline 4. Fam et al. (24) & $\begin{array}{l}20 \mathrm{MDD} \\
20 \mathrm{HC}\end{array}$ & $\begin{array}{l}44.5(9.8) \\
43.7(9.7)\end{array}$ & $\begin{array}{l}\text { (1) PERG } \\
\text { Check size: } 0.8^{\circ} \\
\text { Reversal rate: } 12 \mathrm{rps} \\
\text { Contrasts: } 7,21,42,56,68 \% \\
\text { (2) ffERG: flashes } 0.01 / 3.0 \mathrm{~cd} \cdot \mathrm{s} / \mathrm{m}^{2} \text { ) } \\
\text { (3) Contrast sensitivity (FrACT) }\end{array}$ & $\begin{array}{l}\text { Normal signals in MDD for } \\
\text { (1) PERG contrast gain and } \\
\text { (2) ffERG; } \\
\text { (3) Reduced contrast sensitivity } \\
\text { in MDD which were correlated } \\
\text { with BDl-symptoms }\end{array}$ \\
\hline
\end{tabular}

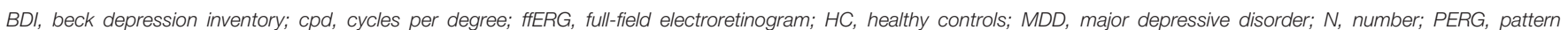
electroretinogram; rps, reversals per second; $S D$, standard deviation.

disorder (MDD) were recruited. PERG measurement was performed within the first few weeks after starting antidepressant medication, without clinical response. The intake of neuroleptics, methylphenidate, or the antidepressant bupropion were defined as exclusion criteria.

In addition, 17 healthy controls without current or a history of psychiatric or neurological diseases were recruited. They had to score within the normal range of the Beck Depression Inventory [BDI; (29)] and the Hamilton Depression Rating Scale [HDRS; (30)]. The matching procedure controlled for effects of sex and age.

Exclusion criteria for both groups were defined as an age $>65$ years, the presence of neurological or ophthalmological diseases or an uncorrectable low visual acuity $(<0.8)$.

The following questionnaires were collected from both patients and control participants: the Beck Depression Inventory [BDI; (29)] to assess the severity of depressive symptoms and the Wender-Utah Rating Scale [WURS-k; (31)] for ADHD symptoms in childhood. In addition, the Hamilton Depression Rating Scale [HDRS; (30)] was applied as third-party assessment questionnaire.

\section{Data Acquisition}

Before examination, visual acuity of each participant was assessed monocularly with the Freiburg Visual Acuity and Contrast Test [FrACT; (32)] and, if necessary, corrected with refraction. A minimum of 0.8 decimal visual acuity was required for each eye.

DTL (Dawson, Trick, and Litzkow)-like electrodes (33), placed at the lower limbus of each eye were used for PERG recordings. Gold-cup electrodes positioned at each ipsilateral eye-canthus served as reference, an ear-clip as ground.

The EP2000 system was used for stimulation and initial data collection (https://michaelbach.de/sci/stim/ep2000/index. html; retrieval date 15.10.2021). Pattern stimuli were presented at an observer distance of $57 \mathrm{~cm}$ on a CRT monitor with $75 \mathrm{~Hz}$ frame rate in $800 \times 600$ pixel resolution, covering a field size of $32^{\circ} \times 27^{\circ}$. Symmetrical black/white reversal checkerboards with a mean luminance of $45 \mathrm{~cd} / \mathrm{m}^{2}$ and a Michelson contrast of $80 \%$ served as pattern stimuli. Four different check sizes $\left(0.8^{\circ}, 1.6^{\circ}, 3.2^{\circ}, 16^{\circ}\right)$ were presented using two different reversal frequencies (12.5 and $18.75 \mathrm{rps}$ ) in the steady state range. Every check size was presented for a duration of 10 sweeps with a constant sweep length for both frequencies $(960 \mathrm{~ms})$, starting with the lower reversal rate, followed by the higher one. Blocks for the different pattern sizes were shown in ascending manner (stepwise increasing check size: $0.8^{\circ}, 1.6^{\circ}, 3.4^{\circ}, 16^{\circ}$ ). This sequence was repeated in 10 equal cycles, with a short break in between. Responses exceeding a threshold of $120 \mu \mathrm{V}$ were automatically rejected as artifacts. A minimum of 100 artifact free sweeps were recorded per condition and submitted to stimulussynchronized averaging.

\section{Data Analysis}

First off-line data processing was performed in Igor Pro 7 (Wave Metrics) with the "EP2000" module. To eliminate mains hum artifacts, averaged response traces were digitally low pass filtered $(40 \mathrm{~Hz})$. A Fourier analysis was performed after any linear trend (e.g., due to baseline drifts) had been removed (34). PERG amplitudes were extracted from the Fourier spectra at the respective stimulation frequencies (12.5 and $18.75 \mathrm{~Hz}$ ) and noise-corrected [as described in (34)]. The average magnitude from the direct adjacent frequencies served as noise estimate (35). Additionally, phases were extracted from the Fourier transformation.

\section{Statistical Analysis}

Statistical analysis was carried out in "R" (36) with RStudio (37) using the "tidyverse" package (38) for data handling. For the 
TABLE 2 | Demographic and psychometric data.

\begin{tabular}{|c|c|c|c|c|}
\hline & Characteristic & Controls, $N=12$ & Patients, $N=12$ & $p$-value ${ }^{a}$ \\
\hline \multirow[t]{2}{*}{ Sex } & Male & $5 / 12$ (42\%) & $5 / 12$ (42\%) & \\
\hline & Female & 7/12 (58\%) & 7/12 (58\%) & \\
\hline \multirow[t]{2}{*}{ Age } & Mean (SD) & $29(8)$ & $26(9)$ & 0.087 \\
\hline & Range & $20-51$ & $19-51$ & \\
\hline \multirow[t]{2}{*}{ Medication } & Medicated & 0/12 (0\%) & 11/12 (92\%) & \\
\hline & Unmedicated & 12/12 (100\%) & 1/12 (8.3\%) & \\
\hline \multirow[t]{2}{*}{ WURS-k } & Mean (SD) & $11(6)$ & 23 (13) & 0.003 \\
\hline & Median (IQR) & $8(7,15)$ & $18(16,27)$ & \\
\hline \multirow[t]{3}{*}{$\mathrm{BDI}$} & Mean (SD) & $3(3)$ & $25(8)$ & $<0.001$ \\
\hline & Median (IQR) & $3(1,5)$ & $25(18,32)$ & \\
\hline & (Missing) & 0 & 1 & \\
\hline \multirow[t]{2}{*}{ HDRS } & Mean (SD) & $1(1)$ & $22(4)$ & $<0.001$ \\
\hline & Median (IQR) & $1(0,1)$ & $22(20,26)$ & \\
\hline
\end{tabular}

a Statistical test: Wilcoxon rank-sum test. BDI, Beck Depression Inventory; HDRS, Hamilton Depression Rating Scale; IQR, interquartile range; $N$, number; SD, standard deviation; WURS-k, Wender-Utah Rating Scale; $y$, years.

8 stimulus conditions ( 4 check sizes and 2 frequencies) PERG amplitudes from both eyes were averaged for every participant separately. Psychometric data comparisons and initial testing for differences between the groups or stimulation parameters were established using Wilcoxon rank sum tests ["rstatix" package (39)]. Response times (in ms) were calculated from the extracted phase values (40). The fully crossed factorial design was analyzed with a mixed analysis of variance (ANOVA) for repeated measures ["afex" package (41)]. The factors group, check size and stimulation frequency, as well as their interactions, were evaluated for their impact on PERG amplitudes or response times. The factors check size and frequency were considered as repeated measures factors for each subject. Post-hoc analysis was limited to group comparisons ["emmeans" package (42)] with equal variance assumed. Hedge corrected (43) Cohen's d was calculated as effect size estimation for unpaired samples ["rstatix" package (39)]. Significance levels were determined by applying the Bonferroni-Holm procedure for a familywise $\alpha$ of 0.05 (44).

\section{RESULTS}

\section{Demographic and Psychometric Data}

Of the originally measured 17 patients, five had to be excluded. The reasons for exclusion were intolerance of the electrodes, the intake of neuroleptic medication, regular somatic medication, subsequently diagnosed psychiatric comorbidity and substance abuse. Finally, 12 patients between 19 and 51 years of age could be included in the final analysis. Four patients suffered from a first severe depressive episode, while 8 patients had a recurrent severe depressive episode. Of the 17 control participants measured, 12 were matched by sex and age to the included patients and considered in the final analysis. The psychometric data of the patient and the control groups are presented in Table 2.

\section{Pattern Electroretinogram (PERG) Group Averaged PERG Responses}

In both groups, measures of one eye of each of two participants had to be excluded due to electrode displacement during the experiment. Except for these cases, the responses of both eyes were averaged before further analysis.

Figure 1 illustrates the mean PERG amplitudes for patients and controls for all stimulus conditions.

The overall average of the PERG response of patients suffering from MDD was significantly lower compared to the control group ( $p=0.017$, unpaired, one-sided Wilcoxon test assuming lower PERG for MDD; data pooled across stimulus parameters), with a similar signal to noise ratio for both groups $(p=$ 0.242 , unpaired, two-sided Wilcoxon test, data averaged across stimulus parameters).

Although the average PERG amplitude in response to the higher frequency (18.75 rps) was significantly lower $(p<0.001$; paired, one-sided Wilcoxon test assuming lower PERG at 18.75 rps; data averaged across groups and check sizes), the signal-to-noise ratio was comparable for both frequencies ( $p=0.121$; paired, two-sided Wilcoxon test, data pooled across groups and check sizes). Further visual inspection suggests largest amplitudes in response to the smallest check size with a slight attenuation toward coarser patterns.

The overall average of PERG response time was significantly reduced in patients with MDD compared to healthy controls $(p$ $<0.001$, unpaired, two-sided Wilcoxon test, data pooled across stimulus parameters).

\section{Mixed ANOVA Results}

With a mixed ANOVA, PERG amplitudes were evaluated for (between) group differences and influences from check size or stimulation frequency, both considered as subject-wise repeated measures (within). Possible interaction effects were included.

The ANOVA revealed a significant effect for the between factor group $\left[F_{(1,22)}=6.53, p=0.018\right]$ and the within factors check size $\left[F_{(1.52,33.41)}=55.42, p<0.001\right]$ and stimulation frequency $\left[F_{(1,22)}=53.02, p<0.001\right]$, as well as a significant interaction between the two stimulus parameters $\left[F_{(1.79,39.38)}=\right.$ 19.24, $p<0.001$ ] on the PERG amplitudes. Interactions between stimulus conditions and the factor group were not observed.

A separate ANOVA calculated for response times showed significant effects for the factors group $\left[F_{(1,22)}=14.70, p\right.$ $<0.001]$, frequency $\left[F_{(1,22)}=88.14, p<0.001\right]$ and size $\left[F_{(1.48,32.51)}=1313.83, p<0.001\right]$.

\section{Post-hoc Analysis for Group Differences}

A subsequent post-hoc comparison of the two groups indicated significantly reduced PERG amplitudes in the MDD group for almost all stimulus parameters (Table 3), considering the uncorrected results. After correcting significance levels for multiple comparisons according to the Bonferroni-Holm procedure, group differences remained significant only for the finest pattern $\left(0.8^{\circ}\right)$. At $18.75 \mathrm{rps}$, the decline of PERG in MDD (Table 3) apparently scales with the check size of the stimulus. 


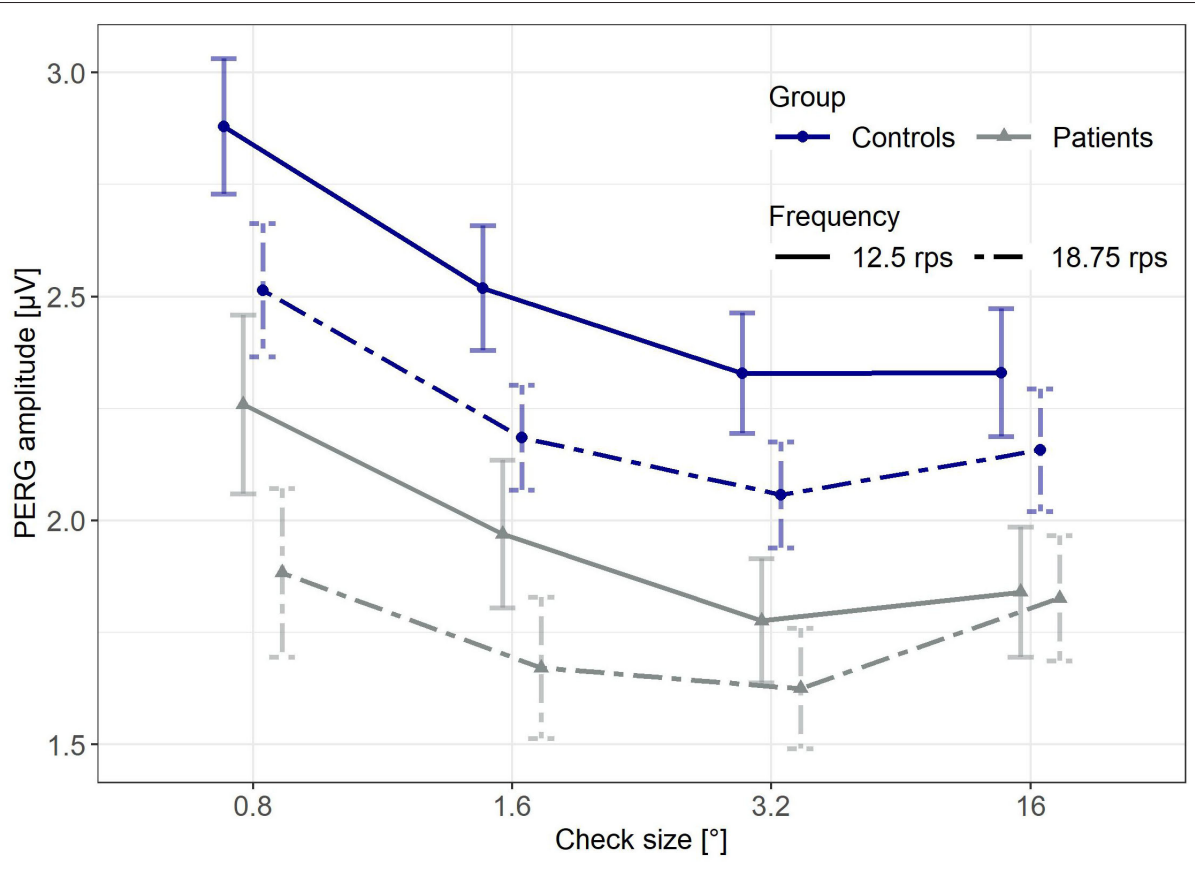

FIGURE 1 | Mean PERG amplitudes for both groups and all stimulus conditions. Error bars indicate the standard error of the mean (SE).

TABLE 3 | Results from the post-hoc analysis for PERG amplitudes (in $\mu$ V).

\begin{tabular}{|c|c|c|c|c|c|c|}
\hline Check Size $\left[^{\circ}\right]$ & Frequency [rps] & Controls mean (SD) & Patients mean (SD) & $\begin{array}{l}\text { Differences of estimated } \\
\text { marginal means (SE) }\end{array}$ & $\begin{array}{l}P \text {-value (significance } \\
\text { level Holm adjusted) }\end{array}$ & $\begin{array}{l}\text { Hedge corrected } \\
\text { Cohen's d }\end{array}$ \\
\hline 0.8 & 12.5 & $2.9(0.5)$ & $2.3(0.7)$ & $0.621(0.21)$ & $0.007\left(^{\star}\right)$ & 0.98 \\
\hline 3.2 & 12.5 & $2.3(0.5)$ & $1.8(0.5)$ & $0.553(0.21)$ & 0.014 (ns) & 1.13 \\
\hline 16 & 12.5 & $2.3(0.5)$ & $1.8(0.5)$ & $0.490(0.21)$ & 0.028 (ns) & 0.95 \\
\hline 3.2 & 18.75 & $2.1(0.4)$ & $1.6(0.5)$ & $0.431(0.21)$ & 0.051 (ns) & 0.95 \\
\hline 16 & 18.75 & $2.2(0.5)$ & $1.8(0.5)$ & $0.331(0.21)$ & 0.127 (ns) & 0.67 \\
\hline
\end{tabular}

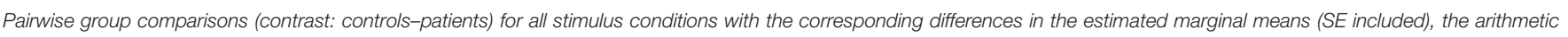

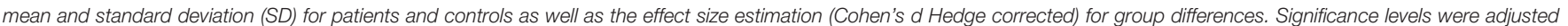
according to Bonferroni-Holm procedure. *significant; ns, not significant.

In an additional post-hoc analysis, we detected shorter response times for patients with MDD compared to healthy controls for all stimulus parameter combinations (Table 4).

\section{Cohen's d Effect Size Estimation for Stimulus Parameter Combinations}

Figure 2 shows that, at a stimulation frequency of 18.75 rps, the PERG response difference between patients and healthy controls is gradually smaller with increasing check size. The most prominent decay in patients' PERG amplitudes was observed with the finest pattern $\left(0.8^{\circ}\right)$ (25\%, $d=1.04)$, while the PERG amplitudes at the coarsest checkerboard $\left(16^{\circ}\right)$ seemed to be least affected (15\%, $d$ $=0.67)$. Interestingly, this PERG response pattern is reminiscent of the conditions observed in early glaucoma $(16,17,45)$.

\section{PERG Check Size Ratio}

Based on the "PERG ratio protocol" in early glaucoma $(17,46$, 47), check size ratios for the PERG amplitudes were established for both groups and frequencies according to formula (1).

$$
\text { PERG ratio }=\frac{\text { PERG amplitude at } 0.8^{\circ}}{\text { PERG amplitude at } 16^{\circ}} .
$$

With regard to the application of PERG response as an objective biomarker, the calculation of PERG ratios for every subject has the advantage of minimizing inter-individual 
TABLE 4 | Results from the post-hoc analysis for calculated response times (in ms).

\begin{tabular}{|c|c|c|c|c|c|c|}
\hline Check size $\left[^{\circ}\right]$ & Frequency [rps] & Controls mean (SD) & Patients mean (SD) & $\begin{array}{l}\text { Differences of estimated } \\
\text { marginal means (SE) }\end{array}$ & $\begin{array}{l}P \text {-value (significance } \\
\text { level Holm adjusted) }\end{array}$ & $\begin{array}{l}\text { Hedge corrected } \\
\text { Cohen's d }\end{array}$ \\
\hline 0.8 & 12.5 & $55.2(2.9)$ & 51.9 (3) & $3.37(0.96)$ & $0.002\left({ }^{*}\right)$ & 1.09 \\
\hline 3.2 & 12.5 & $48.9(2.2)$ & $45.3(2.4)$ & $3.58(0.96)$ & $0.001\left(^{*}\right)$ & 1.53 \\
\hline 16 & 12.5 & $44.7(2.1)$ & $41.5(2.4)$ & $3.22(0.96)$ & $0.002\left(^{*}\right)$ & 1.36 \\
\hline 3.2 & 18.75 & $50.4(2.1)$ & 46.5 (2) & $3.87(0.96)$ & $<0.0001\left(^{*}\right)$ & 1.84 \\
\hline 16 & 18.75 & $46.5(2.1)$ & $43(1.8)$ & $3.52(0.96)$ & $0.001\left({ }^{*}\right)$ & 1.72 \\
\hline
\end{tabular}

Pairwise group comparisons (contrast: controls-patients) for all stimulus conditions with the corresponding differences in the estimated marginal means (SE included), the arithmetic

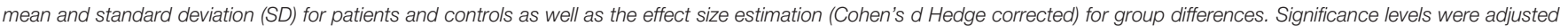
according to Bonferroni-Holm procedure. *significant; ns, not significant.

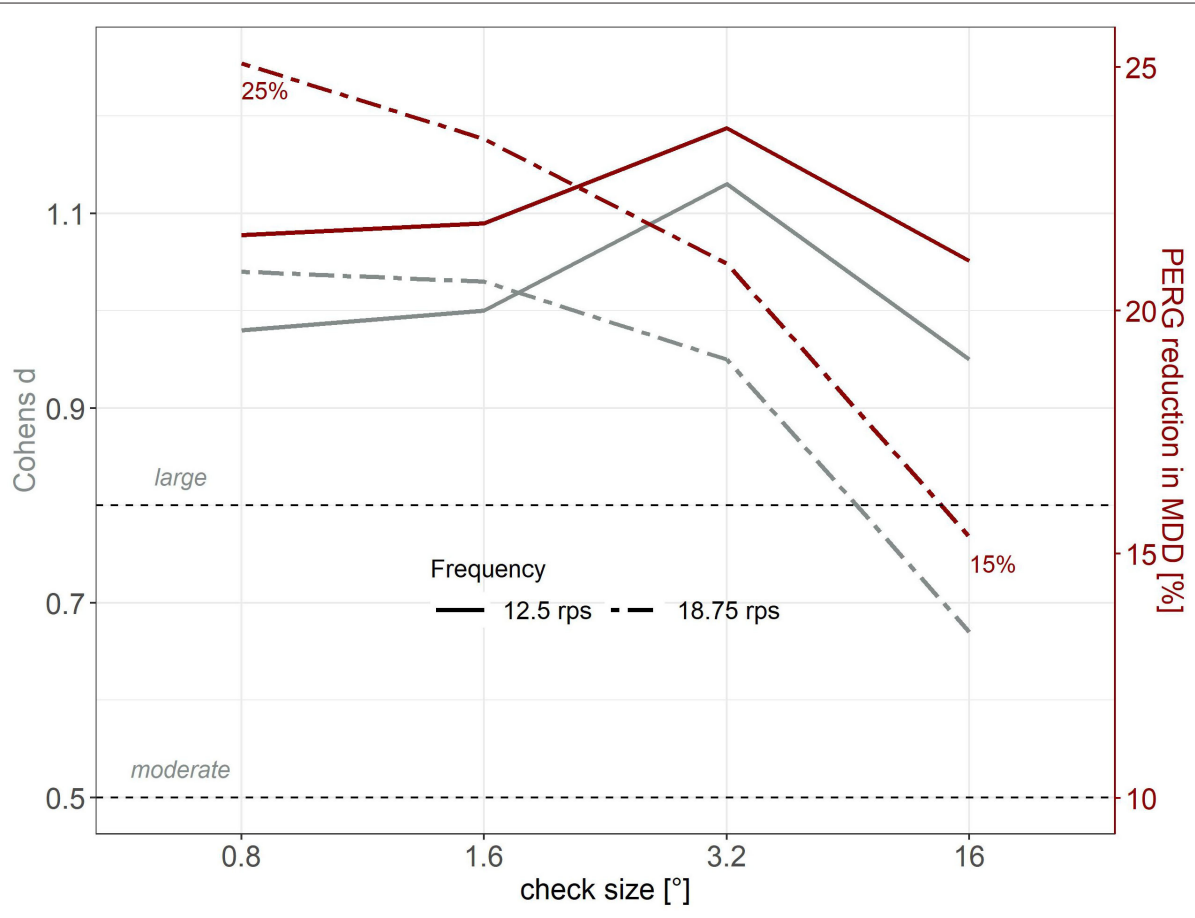

FIGURE 2 | Cohen's d (gray, left axis) as effect size estimation and mean relative PERG reduction (red, right axis, \%) in MDD patients compared to healthy controls as a function of check size. Continuous lines depict 12.5 rps, dashed lines represent 18.75 rps.

variability by amplitude normalization. Figure 3 depicts the normalized PERG amplitudes for the patient and the control group.

A second mixed ANOVA with the between factor group, the within-factor stimulation frequency and the PERG ratio as dependent variable revealed a significant influence of stimulus frequency on the PERG ratio $\left[F_{(1,22)}=29.23, p<0.001\right]$, no overall-group differences $\left[F_{(1,22)}=3.33, p=0.082\right]$, but a significant interaction effect between group and stimulus frequency $\left[F_{(1,22)}=6.96, p=0.015\right]$.

Post-hoc evaluation exhibited that the PERG ratios in the MDD group, in response to a stimulation frequency of $18.75 \mathrm{rps}$, were significantly reduced ( $p=0.008, d=1.07$ ), whereas with the lower reversal rate (12.5 rps), PERG ratios did not differ between groups $(p=0.674, d=0.18)$.

\section{DISCUSSION}

The aims of the present study were (1) the replication of the PERG amplitude effect in patients with MDD and (2) the evaluation of different stimulus conditions to further improve PERG recording procedures for this patient group. Four check sizes $\left(0.8^{\circ}, 1.6^{\circ}, 3.2^{\circ}\right.$, and $\left.16^{\circ}\right)$ were compared to analyze if PERG signals in $\mathrm{MDD}$, in response to coarser patterns, are affected to the same extent as to smaller check 


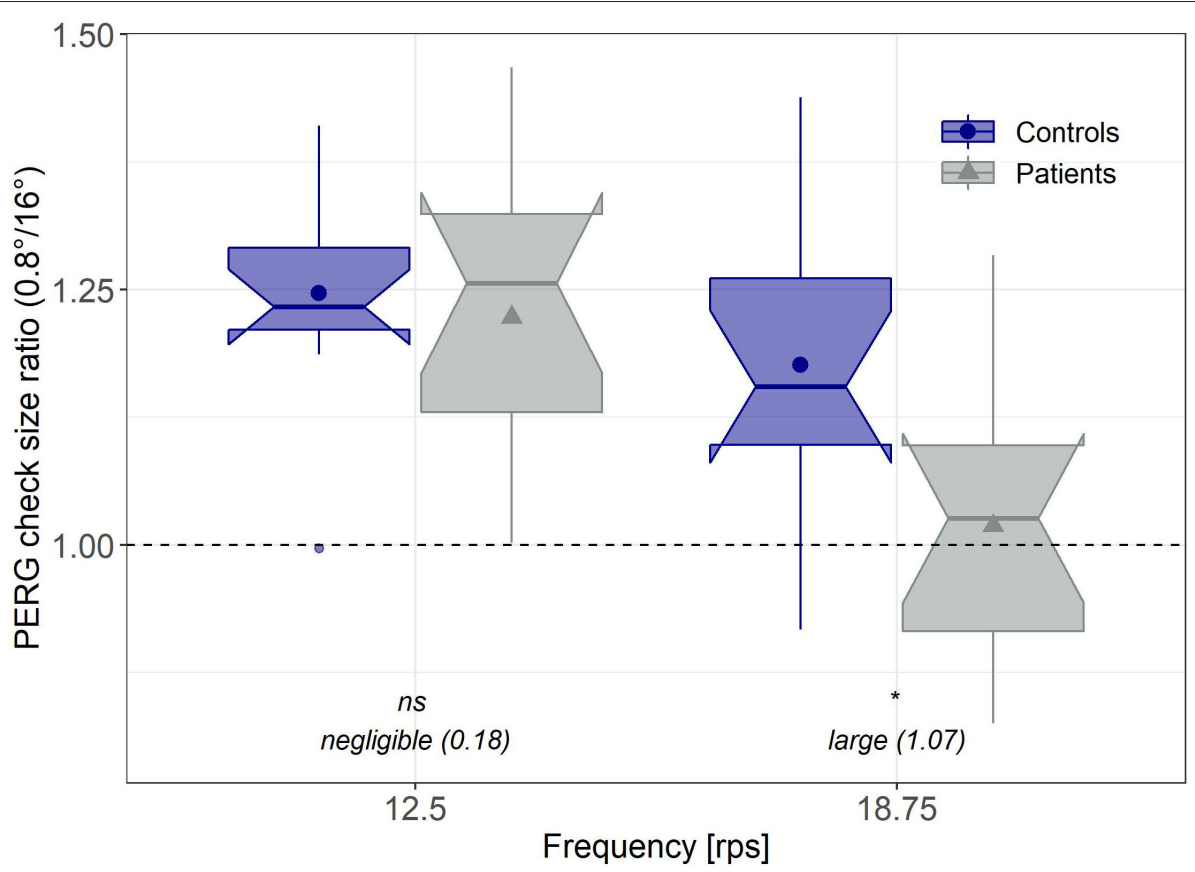

FIGURE 3 | Normalized PERG amplitudes. Individual PERG ratios $\left(0.8^{\circ} / 16^{\circ}\right)$ corresponding to the "PERG ratio protocol" for early glaucoma (17) for both groups and frequencies. Significance levels were Bonferroni-Holm corrected. Effect sizes were estimated based on Hedge's corrected Cohen's d. *significant; ns, not significant.

sizes used in previous studies. The application of coarser patterns would be beneficial by eliminating influences due to refraction errors. In addition, two frequencies (12.5 and $18.75 \mathrm{rps}$ ) for checkerboard reversals were investigated in order to test if PERG responses were similarly affected at higher stimulation frequencies.

\section{Group Comparisons for the Different Parameter Combinations}

Overall, we discerned smaller PERG amplitudes in patients with MDD compared to matched healthy control participants, which replicates earlier findings with an independent sample of patients and controls (9). After correction for multiple testing, statistically reliable reductions in the PERG in MDD were only indicated with the smallest check size $\left(0.8^{\circ}\right)$.

This effect was not only present for the lower (12.5 rps), as previously reported $(9,10)$, but also for the higher stimulation frequency (18.75 rps) with a comparable signal-to-noise ratio for both frequencies. A follow-up study should address, whether a higher frequency can provide the opportunity to reduce total recording time. A shorter measurement time would be particularly advantageous for psychiatric patients with depressive symptoms and limited ability to uphold attention.

The higher rate for checkerboard reversals (18.75 rps) is additionally beneficial by fine-tuning group differences between MDD and control subjects through check size dependent modulations, which allow for a normalization of PERG responses via the calculation of check size ratios, similar to those used for the detection of early glaucoma (17).
Besides, our observations are not in line with the results of Fam et al. (24), who reported normal PERG contrast gain in MDD patients applying a stimulation frequency of $12 \mathrm{rps}$ and the check size of $0.8^{\circ}$. This could possibly be due to differences in the technical implementation of the measurement protocol.

Since the PERG amplitude reduction in patients with MDD was only significant with the smallest check size $\left(0.8^{\circ}\right)$, we cannot recommend a recording paradigm which uses exclusively larger check sizes $\left(1.6^{\circ}, 3.2^{\circ}\right.$, or $\left.16^{\circ}\right)$ in the context of psychiatric disorders, which would render the correction of refractive anomalies unnecessary.

Considering the dopamine-dependent regulation of the receptive field sizes in the retina (48) and the assumption of a disturbed dopamine homeostasis in $\operatorname{MDD}(49,50)$, check size specific PERG alterations in MDD patients seem convincing. Particularly, dopamine is known for its modulatory role in the light adaptation of the retina, favoring daylight vision with high acuity, a mechanism provided by the decoupling of horizontal cells in the retina, thereby shrinking the antagonistic surround structures of the receptive fields (48). A disturbed dopamine homeostasis probably results in alterations in the PERG signals in response to different check sizes as it was similarly described for patients suffering from Parkinson's disease $(4,5)$.

Moreover, shortened response times were observed in patients with MDD for all stimulus conditions. As described in transient stimulations (51), the effect of a shorter response time with larger check sizes is observed in both groups. In patients with glaucoma, not only reduced amplitudes but also shorter response 
times have been similarly described by Bode and colleagues (40). At this point, the authors discuss an effect observed by Viswanathan et al. (52) that leads to a shortening of the P50 peak time when N95 is eliminated. Whether such a differential change between the N95 and the P50, which both contribute to the PERG signal, occurs in MDD and can explain the observed changes in the steady-state response time would need to be addressed in future studies.

\section{PERG Ratio}

While it would have been useful to find strong effects of depression with the very large check sizes, which would have obviated refraction, one can turn the relative constancy of these amplitudes into our favor by using them for individual normalization. Inspired by the PERG ratio protocol in early glaucoma $(16,17,45)$, we compared the standardized PERG amplitudes, i.e., the amplitude ratio over the two check sizes $\left(0.8^{\circ}\right.$ and $\left.16^{\circ}\right)$, between groups for both stimulation frequencies. The advantage of this "PERG ratio" approach is that it reduces inter-individual variability. We observed a significantly reduced PERG ratio in MDD patients compared to healthy controls for the high stimulation frequency (18.75 rps), but not for the low stimulation frequency (12.5 rps). This alternative analysis approach is promising since it increases interpersonal comparability and statistical power, which is particularly important for an objective biomarker. Moreover, higher stimulation frequencies could possibly reduce the time required for recording, which should be addressed in a followup study.

\section{Methodological Issues and Limitations}

The present study provides promising perspectives for the optimization of PERG recording procedures in psychiatric settings. However, some limitations have to be mentioned.

Due to the small number of patients, the results of the current study must be considered preliminary. Follow-up studies with larger samples sizes could yield further information about the adaptation of stimulation frequency and check size for PERG recordings in psychiatric patients. It should be noted, however, that the PERG ratio is feasible to minimize interindividual differences.

At the time of measurement, patients had already been taking antidepressant medication for a few days or weeks. In a previous study by Bubl et al. (9), however, a reduction in contrast gain was detected in both medicated and un-medicated depressed patients. Another limitation is that smoking status was not considered as a matching factor between patients and controls. This could also have a confounding effect on results, as it could have an impact on dopamine neurotransmission (53). Lastly, the MDD group also exhibited elevated ADHD symptoms in childhood according to the WURS-k questionnaire compared to the control group. Since the PERG amplitudes from patients suffering from ADHD have been reported to be unaffected (54), we regard influences from ADHD symptoms as rather unlikely. Particularly since the so-called PERG noise, which has been shown to be elevated in ADHD patients (55), was not affected in our MDD patients $(p=0.94$, one-sided Wilcoxon test comparing PERG noise between groups, data pooled across stimulus parameters).

\section{Summary}

In summary, in this methodological pilot study we could reproduce earlier findings of reduced PERG amplitudes in patients with depression as a potentially objective biomarker signal of the essentially subjective state of depression. In addition, we were able to methodologically improve the recording procedure by demonstrating the suitability of a higher stimulation frequency for recordings along with the introduction of an interpersonal normalization approach for the PERG signals, which further enhances the sensitivity of the method.

\section{DATA AVAILABILITY STATEMENT}

The original contributions presented in the study are included in the article, further inquiries can be directed to the corresponding author/s.

\section{ETHICS STATEMENT}

The studies involving human participants were reviewed and approved by the Ethics Committee of the University Medical Center Freiburg (Approval ID: 93/04). The patients/participants provided their written informed consent to participate in this study.

\section{AUTHOR CONTRIBUTIONS}

$\mathrm{KN}$ and $\mathrm{EF}$ wrote the paper. EF, KN, CS, and SM performed the data and statistical analysis. LTvE, KN, DEb, MB, and EF organized the study and created the study design. $\mathrm{KN}, \mathrm{DEb}$, and DEn recruited the patients and established the diagnosis. MB and $\mathrm{SH}$ supported the methodological and technical realization for the collection of the electrophysiological data. CS and EF performed the measurements. LTvE, KD, SM, DEb, DEn, $\mathrm{KR}, \mathrm{EB}, \mathrm{MB}, \mathrm{JK}$, and $\mathrm{SH}$ revised the manuscript critically focusing on clinical and statistical aspects. All authors were critically involved in the theoretical discussion, composition of the manuscript, and read and approved the final version of the manuscript.

\section{FUNDING}

Part of the study was funded by the DFG (HE 3504/11-1 | TE 280/24-1). The article processing charge was funded by the Baden-Wuerttemberg Ministry of Science, Research and Art and the University of Freiburg in the funding programme Open Access Publishing. 


\section{REFERENCES}

1. Nguyen CTO, Hui F, Charng J, Velaedan S, van Koeverden AK, Lim JKH, et al. Retinal biomarkers provide 'insight' into cortical pharmacology and disease. Pharmacol Therapeut. (2017) 175:151-77. doi: 10.1016/j.pharmthera.2017.02.009

2. Almonte MT, Capellàn P, Yap TE, Cordeiro MF. Retinal correlates of psychiatric disorders. Therapeut Adv Chronic Dis. (2020) 11:1-21. doi: $10.1177 / 2040622320905215$

3. Tebartz van Elst L, Greenlee MW, Foley JM, Lücking CH. Contrast detection, discrimination and adaptation in patients with Parkinson's disease and multiple system atrophy. Brain J Neurol. (1997) 120(Pt 12):2219-28.

4. Brandies R, Yehuda $S$. The possible role of retinal dopaminergic system in visual performance. Neurosci Biobehav Rev. (2008) 32:611-56. doi: 10.1016/j.neubiorev.2007.09.004

5. Armstrong RA. Visual symptoms in Parkinson's disease. Parkinsons Dis. (2011) 2011:e908306. doi: 10.4061/2011/908306

6. Silverstein S, Keane BP, Blake R, Giersch A, Green M, Kéri S. Vision in schizophrenia: why it matters. Front Psychol. (2015) 6:41. doi: 10.3389/fpsyg.2015.00041

7. Silverstein SM, Fradkin SI, Demmin DL. Schizophrenia and the retina: towards a 2020 perspective. Schizophrenia Res. (2020) 219:84-94. doi: 10.1016/j.schres.2019.09.016

8. Bubl E, Tebartz van Elst L, Gondan M, Ebert D, Greenlee MW. Vision in depressive disorder. World J Biol Psychiatry. (2009) 10 (4 Pt 2):37784. doi: 10.1080/15622970701513756

9. Bubl E, Kern E, Ebert D, Bach M, Tebartz van Elst L. Seeing gray when feeling blue? Depression can be measured in the eye of the diseased. Biol Psychiatry. (2010) 68:205-8. doi: 10.1016/j.biopsych.2010.02.009

10. Bubl E, Ebert D, Kern E, Tebartz van Elst L, Bach M. Effect of antidepressive therapy on retinal contrast processing in depressive disorder. Brit J Psychiatry. (2012) 201:151-8. doi: 10.1192/bjp.bp.111.100560

11. Robson AG, Nilsson J, Li S, Jalali S, Fulton AB, Tormene AP, et al. ISCEV guide to visual electrodiagnostic procedures. Document Ophthalmol. (2018) 136:1-26. doi: 10.1007/s10633-017-9621-y

12. Luo $\mathrm{X}$, Frishman LJ. Retinal pathway origins of the pattern electroretinogram (PERG). Investig Ophthalmol Visual Sci. (2011) 52:8571-84. doi: 10.1167/iovs.11-8376

13. Bach M, Hoffmann MB. The origin of the pattern electroretinogram. In: Heckenlively JR, Arden GB, editors. Principles Practice of Clinical Electrophysiology of Vision. 2nd ed. Cambridge, MA: MIT Press (2006), p. 185-96.

14. Bach $M$, Brigell MG, Hawlina $M$, Holder GE, Johnson MA, McCulloch DL, et al. ISCEV standard for clinical pattern electroretinography (PERG): 2012 update. Document Ophthalmol. (2012) 124:1-13. doi: 10.1007/s10633-012-9353-y

15. Holder GE. Pattern electroretinography (PERG) and an integrated approach to visual pathway diagnosis. Prog Retinal Eye Res. (2001) 20:53161. doi: 10.1016/S1350-9462(00)00030-6

16. Bach M. Electrophysiological approaches for early detection of glaucoma. Eur J Ophthalmol Suppl. (2001) 11 (Suppl 2):S419. doi: $10.1177 / 112067210101102$ s05

17. Bach M, Hoffmann MB. Update on the pattern electroretinogram in glaucoma. Optomet Vision Sci. (2008) 85:38695. doi: 10.1097/OPX.0b013e318177ebf3

18. Bach M, Ramharter-Sereinig A. Pattern electroretinogram to detect glaucoma: comparing the PERGLA and the PERG ratio protocols. Document Ophthalmol. (2013) 127:227-38. doi: 10.1007/s10633-013-9412-z

19. Langheinrich $T$, Tebartz van Elst L, Lagrèze WA, Bach $M$, Lücking $\mathrm{CH}$, Greenlee MW. Visual contrast response functions in Parkinson's disease: evidence from electroretinograms, visually evoked potentials and psychophysics. Clin Neurophysiol. (2000) 111:66-74. doi: 10.1016/s1388-2457(99)00223-0

20. Lavoie J, Illiano P, Sotnikova TD, Gainetdinov RR, Beaulieu J-M, Hébert M. The electroretinogram as a biomarker of central dopamine and serotonin: potential relevance to psychiatric disorders. Biological Psychiatry. (2014) 75:479-86. doi: 10.1016/j.biopsych.2012.11.024
21. Lavoie J, Maziade M, Hébert M. The brain through the retina: the flash electroretinogram as a tool to investigate psychiatric disorders. Prog Neuropsychopharmacol Biol Psychiatry. (2014) 48:129-34. doi: 10.1016/j.pnpbp.2013.09.020

22. Schwitzer T, Schwan R, Bubl E, Lalanne L, Angioi-Duprez K, Laprevote $\mathrm{V}$. Looking into the brain through the retinal ganglion cells in psychiatric disorders: a review of evidences. Prog Neuropsychopharmacol Biol Psychiatry. (2017) 76:155-62. doi: 10.1016/j.pnpbp.2017. 03.008

23. Bubl E, Kern E, Ebert D, Riedel A, Tebartz van Elst L, Bach M. Retinal dysfunction of contrast processing in major depression also apparent in cortical activity. Eur Arch Psychiatry Clin Neurosci. (2015) 265:34350. doi: 10.1007/s00406-014-0573-x

24. Fam J, Rush AJ, Haaland B, Barbier S, Luu C. Visual contrast sensitivity in major depressive disorder. J Psychosom Res. (2013) 75:83-6. doi: 10.1016/j.jpsychores.2013.03.008

25. Ben-Shlomo G, Bach M, Ofri R. Temporal and spatial frequencies interact in the contrast transfer function of the pattern electroretinogram. Vision Res. (2007) 47:1992-9. doi: 10.1016/j.visres.2007.04.009

26. Bach M, Mathieu M. Different effect of dioptric defocus vs. light scatter on the pattern electroretinogram (PERG). Document Ophthalmol. (2004) 108:99106. doi: 10.1023/b:doop.0000018415.00285.56

27. Trick GL. Retinal potentials in patients with primary open-angle glaucoma: physiological evidence for temporal frequency tuning deficits. Investig Ophthalmol Visual Sci. (1985) 26:1750-58.

28. Hiss P, Fahl G. [Changes in the pattern electroretinogram in glaucoma and ocular hypertension are dependent on stimulus frequency]. Fortschritte Der Ophthalmologie Zeitschrift Der Deutschen Ophthalmologischen Gesellschaft. (1991) 88:562-65.

29. Beck AT, Ward CH, Mendelson M, Mock J, Erbaugh J. An inventory for measuring depression. Arch Gen Psychiatry. (1961) 4:561-71.

30. Hamilton M. A rating scale for depression. J Neurol Neurosurg Psychiatry. (1960) 23:56-62. doi: 10.1136/jnnp.23.1.56

31. Retz-Junginger P, Retz W, Blocher D, Weijers H-G, Trott E, Wender $\mathrm{PH}$, et al. Wender Utah Rating Scale (WURS-k) Die deutsche Kurzform zur retrospektiven Erfassung des hyperkinetischen Syndroms bei Erwachsenen. Der Nervenarzt. (2002) 73:830-38. doi: 10.1007/s00115-0011215-x

32. Bach M. The Freiburg visual acuity test-automatic measurement of visual acuity. Optometry Vision Sci. (1996) 73:4953. doi: 10.1097/00006324-199601000-00008

33. Dawson WW, Trick GL, Litzkow CA. Improved electrode for electroretinography. Invest Ophthalmol Vis Sci. (1979) 18:988-91.

34. Bach M, Meigen T. Do's and don'ts in Fourier analysis of steady-state potentials. Doc Ophthalmol. (1999) 99:69-82. doi: 10.1023/A:1002648202420

35. Meigen T, Bach M. On the statistical significance of electrophysiological steady-state responses. Doc Ophthalmol. (1999) 98:20732. doi: 10.1023/A:1002097208337

36. R Core Team. R: A Language and Environment for Statistical Computing. Vienna: R Foundation for Statistical Computing (2020). Available online at: https://www.R-project.org/

37. RStudio Team. RStudio: Integrated Development Environment for R. Boston, MA: RStudio, PBC (2020). Available online at: http://www.rstudio.com/

38. Wickham H, Averick M, Bryan J, Chang W, McGowan L, François $\mathrm{R}$, et al. Welcome to the Tidyverse. J Open Source Softw. (2019) 4:1686. doi: 10.21105/joss.01686

39. Kassambara A. Rstatix: Pipe-Friendly Framework for Basic Statistical Tests (2020). Available online at: https://CRAN.R-project.org/package=rstatix

40. Bode SFN, Jehle T, Bach M. Pattern electroretinogram in glaucoma suspects: new findings from a longitudinal study. Investig Opthalmol Visual Sci. (2011) 52:4300. doi: 10.1167/iovs.10-6381

41. Singmann H, Bolker B, Westfall J, Aust F, Ben-Shachar MS. Afex: Analysis of Factorial Experiments (2020). Available online at: https://CRAN.R-project. org/package $=$ afex

42. Lenth RV. Emmeans: Estimated Marginal Means, Aka Least-Squares Means (2020). Available online at: https://CRAN.R-project.org/package= emmeans 
43. Hedges LV, Olkin I. Statistical methods for meta-analysis. In: CHAPTER 5 - Estimation of a Single Effect Size: Parametric and Nonparametric Methods. Orlando, FL: Academic Press (1985). p. 75-106. doi: 10.1016/C2009-0-03396-0

44. Holm S. A simple sequentially rejective multiple test procedure. Scand $J$ Statist. (1979) 6:65-70.

45. Bach M, Unsoeld AS, Philippin H, Staubach F, Maier P, Walter HS, et al. Pattern ERG as an early glaucoma indicator in ocular hypertension: a longterm, prospective study. Investig Ophthalmol Visual Sci. (2006) 47:48817. doi: $10.1167 /$ iovs.05-0875

46. Poloschek CM, Bach M. [Electrophysiological examination methods in glaucoma diagnostics]. Der Ophthalmologe: Zeitschrift Der Deutschen Ophthalmologischen Gesellschaft. (2012) 109:35863. doi: 10.1007/s00347-012-2546-7

47. Anders L-M, Heinrich SP, Lagrèze WA, Joachimsen L. Little effect of $0.01 \%$ atropine eye drops as used in myopia prevention on the pattern electroretinogram. Document Ophthalmol. (2019) 138:85-95. doi: 10.1007/s10633-019-09671-0

48. Roy S, Field GD. Dopaminergic modulation of retinal processing from starlight to sunlight. J Pharmacol Sci. (2019) 140:8693. doi: 10.1016/j.jphs.2019.03.006

49. Ebert D, Lammers C-H. Das zentrale dopaminerge System und die Depression. Der Nervenarzt. (1997) 68:545-55. doi: 10.1007/s001150050159

50. Dunlop BW, Nemeroff CB. The role of dopamine in the pathophysiology of depression. Arch Gen Psychiatry. (2007) 64:327. doi: 10.1001/archpsyc.64.3.327

51. Bach M, Holder GE. Check size tuning of the pattern electroretingoram: a reappraisal. Document Ophthalmol. (1996) 92:193-202. doi: 10.1007/BF02583290

52. Viswanathan S, Frishman LJ, Robson JG. The uniform field and pattern ERG in macaques with experimental glaucoma: removal of spiking activity. Investig Ophthalmol Visual Sci. (2000) 41:2797-810.

53. Dani JA. Roles of dopamine signaling in nicotine addiction. Mol Psychiatry. (2003) 8:255-6. doi: 10.1038/sj.mp.4001284
54. Bubl E, Dörr M, Philipsen A, Ebert D, Bach M, Tebartz van Elst L. Retinal contrast transfer functions in adults with without ADHD. PLoS ONE. (2013) 8:e61728. doi: 10.1371/journal.pone. 0061728

55. Werner AL, Tebartz van Elst L, Ebert D, Friedel E, Bubl A, Clement $\mathrm{HW}$, et al. Normalization of increased retinal background noise after ADHD treatment: a neuronal correlate. Schizophr Res. (2019) 219:77-83. doi: 10.1016/j.schres.2019.04. 013

Conflict of Interest: LTvE Advisory boards, lectures, or travel grants within the last three years: Roche, Eli Lilly, Janssen-Cilag, Novartis, Shire, UCB, GSK, Servier, Janssen, and Cyberonics. KD member of the "Steering Committee Neurosciences," Janssen Pharmaceuticals, Inc.

The remaining authors declare that the research was conducted in the absence of any commercial or financial relationships that could be construed as a potential conflict of interest.

Publisher's Note: All claims expressed in this article are solely those of the authors and do not necessarily represent those of their affiliated organizations, or those of the publisher, the editors and the reviewers. Any product that may be evaluated in this article, or claim that may be made by its manufacturer, is not guaranteed or endorsed by the publisher.

Copyright (C) 2021 Friedel, Tebartz van Elst, Schmelz, Ebert, Maier, Endres, Runge, Domschke, Bubl, Kornmeier, Bach, Heinrich and Nickel. This is an open-access article distributed under the terms of the Creative Commons Attribution License (CC $B Y)$. The use, distribution or reproduction in other forums is permitted, provided the original author(s) and the copyright owner(s) are credited and that the original publication in this journal is cited, in accordance with accepted academic practice. No use, distribution or reproduction is permitted which does not comply with these terms. 